\title{
What makes the difference between the natural course of a remaining type $B$ dissection after type $A$ repair and a primary type $B$ aortic dissection? ${ }^{\dagger}$
}

\author{
Eva Krähenbühla ${ }^{\mathrm{a}}$, Sladjana Maksimovica, Gottfried Sodeck ${ }^{\mathrm{b}}$, David Reineke ${ }^{\mathrm{a}}$, Florian Schoenhoffa \\ Jürg Schmidlia, Thierry Carrel ${ }^{\mathrm{a}}$ and Martin Czerny ${ }^{\mathrm{a}, *}$
}

\author{
a Department of Cardiovascular Surgery, University Hospital Berne, Berne, Switzerland \\ b Department of Cardiology, University Hospital Berne, Berne, Switzerland \\ * Corresponding author. Freiburgstrasse 18, 3010 Berne, Switzerland. Tel: +41-31-6322376; fax: +41-31-6322919; e-mail: martin.czerny@insel.ch (M. Czerny).
}

Received 29 September 2011; received in revised form 3 January 2012; accepted 9 January 2012

\begin{abstract}
OBJECTIVES: To analyse the outcome and need for intervention [surgery or thoracic endovascular aortic repair (TEVAR)] in patients after surgery for remaining type B dissection after type A repair and primary type B aortic dissection.

METHODS: Within a 10-year period, 247 patients with remaining type B after type A, and 112 patients with primary type B aortic dissection were analysed. We assessed the clinical outcome as well as the need for intervention (surgery or TEVAR) within the aortic arch and the thoracoabdominal aorta as well as risk factors.

RESULTS: The median follow-up was 23 months (interquartile range 5-52). There was a significant difference with regard to the status of the primary entry tear between patients after surgical repair of an acute type A aortic dissection and primary acute type B aortic dissection (patent vs. non-patent entry 35 vs. 83\%, $P<0.001$ ). The overall need for any kind of intervention (surgery or TEVAR) was $19 \%$. Multivariate Cox regression analysis revealed a patent primary entry tear in patients after surgery for acute type A aortic dissection as an independent predictor for intervention (surgery or TEVAR) during follow-up [odds ratio (OR) 6.4; confidence interval (CI) 1.39-29.81, $P=0.017$. Multivariate Cox regression analysis did not reveal a patent primary entry tear in patients after acute type $B$ aortic dissection as an independent predictor for intervention (surgery or TEVAR) during follow-up (OR 0.67; CI 0.27-1.69, P=0.671). Finally, the thrombosis status of the false lumen was not an independent predictor for intervention (surgery or TEVAR) either in patients after surgery for acute type $\mathrm{A}$ aortic dissection ( $\mathrm{OR} 3.46$; $\mathrm{Cl} 0.79-15.16, \mathrm{P}=0.100$ ) or in patients after acute type $\mathrm{B}$ aortic dissection (OR 0.77; $\mathrm{Cl} 0.31$ 1.93, $P=0.580)$.

CONCLUSIONS: A remaining type B dissection after type A repair and a primary type $\mathrm{B}$ aortic dissection represent two distinct pathophysiological entities with regard to late outcome. The need for any kind of intervention in the thoracoabdominal aorta is significantly higher in primary type B aortic dissections. A remaining patent primary entry tear independently predicts the need for intervention (surgery or TEVAR) in patients after surgery for acute type A aortic dissection and, thereby, remains the main target of initial therapy. The thrombosis status of the false lumen seems to be of secondary importance.
\end{abstract}

Keywords: Type A aortic dissection - Type B aortic dissection • Natural course $\cdot$ Secondary surgical conversion

\section{INTRODUCTION}

The continuing dilation of various segments of the thoracoabdominal aorta in patients after surgical repair of acute type $A$ aortic dissection is frequently seen $[1,2]$. A certain percentage of these patients require thoracoabdominal replacement to various extents [3]. Knowledge regarding the risk factors of this development is limited. The continuing dilation of various segments of the thoracoabdominal aorta in patients after acute type $B$ dissection is also frequently seen [1, 4]. A certain

'Presented at the 25th Annual Meeting of the European Association for Cardio-Thoracic Surgery, Lisbon, Portugal, 1-5 October 2011. percentage of these patients also require thoracoabdominal replacement to various extents [3]. Knowledge regarding the risk factors for this development are better defined and include aortic size as well as thrombosis status of the false lumen [4-6]. Nevertheless, no direct comparison of the natural course of a remaining type $B$ dissection after surgery for type $A$ dissection and the natural course of a primary type $B$ dissection has been made.

The aim of this study was to analyse the outcome and need for surgical intervention in patients after surgery for remaining type $B$ dissection after type $A$ repair and primary type $B$ aortic dissection. 


\section{METHODS}

\section{Patients}

All patients having been discharged after referral for treatment of acute type A (De Bakey type I) or acute type B aortic dissection [thoracic endovascular aortic repair (TEVAR) or conservatively] from our centre between January 2001 and December 2010 were analysed. Prerequisites for inclusion in the present study were a computed tomography (CT) angiography (CTA) or magnetic resonance angiography (MRA) at the time of discharge as well as at least one CTA or four MRA during follow-up. Patient demographics are shown in Table 1. Patients with connective tissue diseases were excluded. Furthermore, patients with acute aortic syndromes such as symptomatic penetrating aortic ulcer, intramural haematoma, as well as traumatic aortic injury were also excluded. Our surgical and perfusional strategy in patients with acute type A aortic dissection has been described in detail previously [7]. From a surgical standpoint, hemiarch replacement was the minimum requirement.

\section{Morphological assessment}

The first CT scan and the last available CTA of each patient were analysed with regard to the status of the primary entry tear (patent vs. non-patent) as well as to the thrombosis status of the false lumen in the proximal and mid-descending segment (perfused, partially thrombosed and fully thrombosed). Patients in whom an exact determination of the status of the primary entry tear could not be evaluated on axial, sagittal or coronary projections in CTA or MRA were not considered for analysis.

\section{Follow-up and need for surgery or intervention}

During follow-up, survival as well as the need for intervention (surgery or TEVAR) within the aortic arch or the thoracoabdominal

Table 1: Descriptive characteristics of the cohort

$n$ overall $=359$

$\begin{array}{lrl}\text { Demographics } & & \\ \text { Age, median (IQR) } & 62 & (53-71) \\ \text { Female, } n(\%) & 82 & (23 \%) \\ \text { Hypertension, } n(\%) & 339 & (94 \%) \\ \text { Diabetes, } n(\%) & 47 & (13 \%) \\ \text { Aortic-related assessment } & & \\ \quad \text { Type A aortic dissection, } n(\%) & 247 & (69 \%) \\ \text { Type B aortic dissection, } n(\%) & 112 & (31 \%) \\ \text { Closure of the primary entry, } n(\%) & 251 & (70 \%) \\ \text { Full thrombosis of false lumen, } n(\%) & 211 & (59 \%) \\ \text { Partial thrombosis of false lumen, } n(\%) & 64 & (18 \%) \\ \text { Outcome } & & \\ \text { Follow-up in months, median (IQR) } & 23 & (5-52) \\ \text { All-cause death during follow-up, median (IQR) } & 24 & (7 \%) \\ \text { Need for reintervention, } n(\%) & 67 & (19 \%) \\ \text { Open surgery, } n \text { (\%) } & 26 & (7 \%)\end{array}$

Unless otherwise indicated, data are number (percentage). IQR: interquartile range; classification of chronic health conditions and risk factors according to EuroSCORE criteria. aorta were assessed. Threshold for surgery or TEVAR was an aortic diameter of at least $5.5 \mathrm{~cm}$ at any level.

\section{Statistical methods}

Continuous data are presented as the median and the interquartile range (IQR) (range from the 25th to the 75th percentile). Discrete data are given as counts and percentages. Comparisons of continuous data were performed by Mann-Whitney U-tests, and groups of categorical data were compared by $\chi^{2}$ tests. Univariate Cox regression analysis was primarily performed to assess the prognostic impact of the primary entry status upon the future need for reinterventions, followed by a multivariate Cox regression to adjust for the thrombosis status of the false lumen. Results of the regression model are given as the odds ratio $(\mathrm{OR})$ and the $95 \%$ confidence interval $(95 \% \mathrm{Cl})$. Regression diagnostics and overall model-fit were performed according to standard procedures. A two-sided $P<0.05$ was considered statistically significant. Calculations were performed with SPSS for Mac OsX (version 19.0).

\section{RESULTS}

\section{Chronic health conditions and risk factors}

The median age of all patients was 62 (IQR 53-71), 23\% were female. Hypertension was diagnosed in $94 \%$ and diabetes mellitus in $13 \%$ (Table 1).

\section{Aortic-related assessment-all patients}

Sixty-nine per cent had the primary diagnosis of an acute type A aortic dissection and $31 \%$ had the primary diagnosis of an acute type B aortic dissection. In $70 \%$, the primary entry tear was nonpatent at the first CTA before discharge. In 59\%, full thrombosis of the false lumen was seen (Table 1).

\section{Aortic-related assessment-patent vs. non-patent primary entry tear}

There was a significant difference with regard to the status of the primary entry tear between patients after surgical repair of an acute type A aortic dissection and primary acute type B aortic dissection (patent vs. non-patent entry 35 vs. $83 \%, P<0.001$ ). Comparable findings were made according to the thrombosis status of the false lumen: full thrombosis of the false lumen (patent vs. non-patent entry 10 vs. 80\%, $P<0.001$ ) and partial thrombosis of the false lumen (patent vs. non-patent entry 24 vs. $15 \%, P=0.04$ ) (Table 2 ).

\section{Follow-up}

Median follow-up was 23 months (IQR 5-52). All-cause death during follow-up was $7 \%$. The overall need for any kind of intervention (surgery or TEVAR) was 19\%. Seven per cent of all patients underwent thoracoabdominal replacement to various 
Table 2: Distribution of patients by different chronic health conditions and aortic-related assessment stratified to entry-state during follow-up

Patent entry $(n=108)$ vs. non-patententry $(n=251)$

$P$-value

\begin{tabular}{lc}
\hline Demographics & \\
Age, median (IQR) & $63(52-71)$ \\
Female sex, $n(\%)$ & $21(19 \%)$ \\
Hypertension, $n(\%)$ & $104(94 \%)$ \\
Diabetes mellitus, $n(\%)$ & $41(38 \%)$ \\
Aortic-related assessment & \\
$\quad$ Type A aortic dissection, $n(\%)$ & $38(35 \%)$ \\
Full thrombosis of the false lumen, $n(\%)$ & $10(10 \%)$ \\
Partial thrombosis of the false lumen, $n(\%)$ & $26(24 \%)$ \\
Outcome & \\
Follow-up in months, median (IQR) & $25(6-55)$ \\
Need for reintervention, $n(\%)$ & $35(32 \%)$ \\
Open surgery, $n(\%)$ & $17(16 \%)$
\end{tabular}

$\begin{array}{cc}62(53-71) & 0.79 \\ 61(24 \%) & 0.31 \\ 235(94 \%) & 0.31 \\ 103(41 \%) & 0.59 \\ & \\ 209(83 \%) & <0.001 \\ 201(80 \%) & <0.001 \\ 38(15 \%) & 0.04 \\ & \\ 23(5-52) & 0.54 \\ 32(13 \%) & <0.001 \\ 9(6 \%) & <0.001\end{array}$

Unless otherwise indicated, data given are in number (percentages).

IQR: interquartile range; classification of chronic health conditions and risk factors according to EuroSCORE criteria.
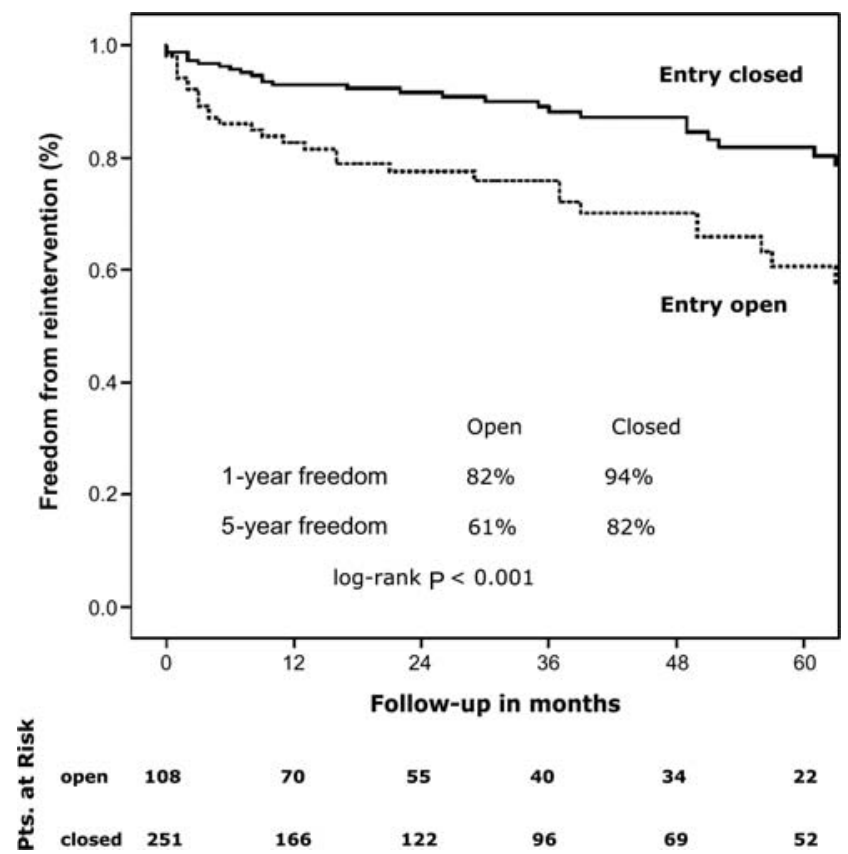

Figure 1: Freedom from intervention (surgery or TEVAR) in the entire patient population according to the status of the primary entry tear.

extents (Table 1). One-year and 5-year freedom from intervention rates in the entire patient population with regard to the status of the primary entry tear (patent vs. non-patent) was 82 and $61 \%$ vs. 94 and 82\%, respectively (log-rank <0.001) (Fig. 1).

One-year and 5-year freedom from intervention rates in patients after surgery for acute type A aortic dissection with regard to the status of the primary entry tear (patent vs. nonpatent) was 83 and $79 \%$ vs. 96 and 90\%, respectively (log-rank = 0.028) (Fig. 2). One-year and 5-year freedom from intervention rates in patients after acute type $B$ aortic dissection with regard to the status of the primary entry tear (patent vs. non-patent)

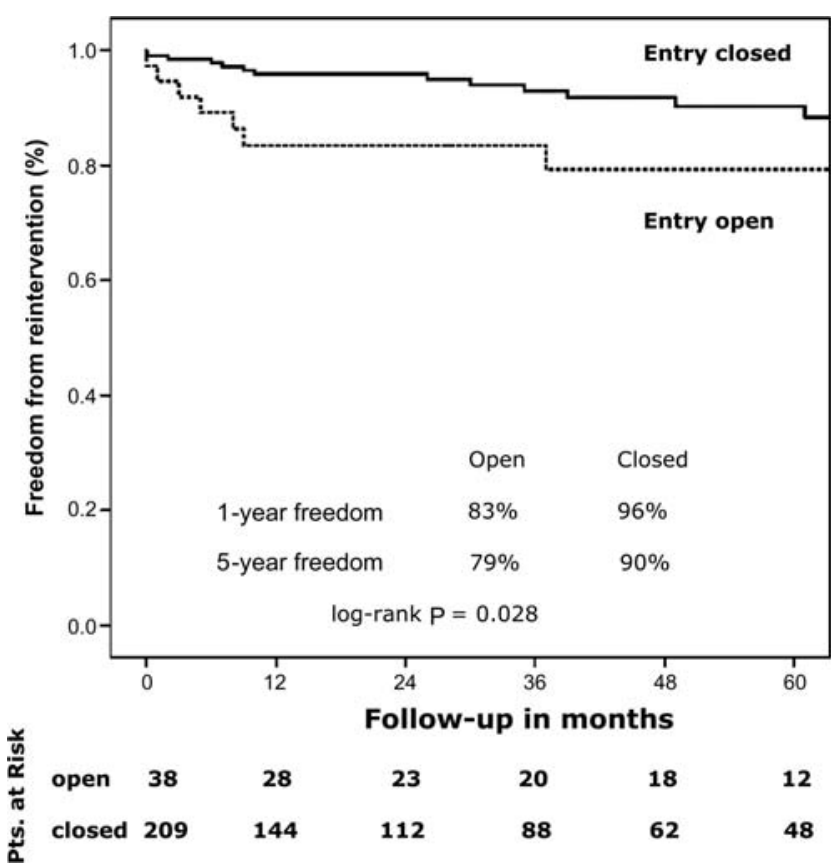

Figure 2: Freedom from intervention (surgery or TEVAR) in patients after surgery for acute type A aortic dissection according to the status of the primary entry tear.

was 82 and $47 \%$ vs. 80 and $35 \%$, respectively (log-rank $=0.51$ ) (Fig. 3).

\section{Univariate Cox regression analysis}

Univariate Cox regression analysis revealed a patent primary entry tear in patients after surgery for acute type A aortic dissection as a predictor for intervention (surgery or TEVAR) during follow-up (OR 2.4; Cl 1.07-5.40, P=0.034). Univariate Cox regression analysis did not reveal a patent primary entry tear in 


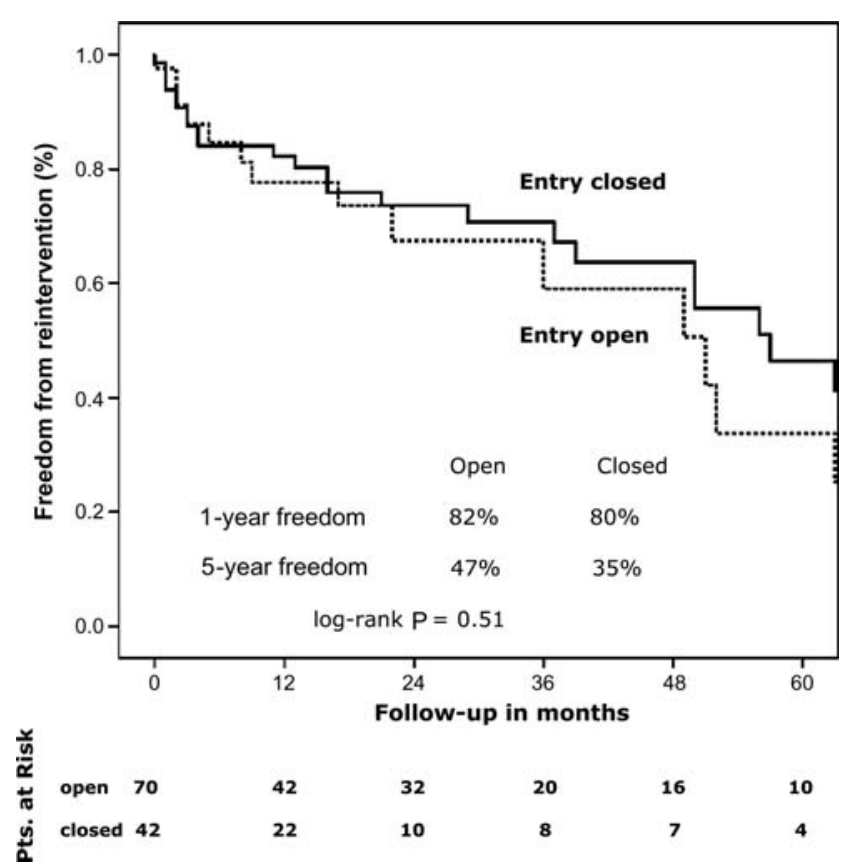

Figure 3: Freedom from intervention (surgery or TEVAR) in patients after acute type $B$ aortic dissection according to the status of the primary entry tear.

patients with acute type B aortic dissection as a predictor for intervention (surgery or TEVAR) during follow-up (OR $0.8 ; \mathrm{Cl}$ $0.46-1.54, P=0.052$ ).

\section{Multivariate Cox regression analysis}

Multivariate Cox regression analysis revealed a patent primary entry tear in patients after surgery for acute type A aortic dissection as an independent predictor for intervention (surgery or TEVAR) during follow-up (OR 6.4; $\mathrm{Cl}$ 1.39-29.81, $P=0.017$ ).). Multivariate Cox regression analysis did not reveal a patent primary entry tear in patients after acute type B aortic dissection as an independent predictor for intervention (surgery or TEVAR) during follow-up (OR $0.67 ; \mathrm{Cl} 0.27-1.69, P=0.671$ ). Finally, the thrombosis status of the false lumen was not an independent predictor for intervention (surgery or TEVAR) either in patients after surgery for acute type A aortic dissection (OR 3.46; CI 0.79$15.16, P=0.100)$ or in patients after acute type $B$ aortic dissection (OR 0.77; Cl 0.31-1.93, $P=0.580$ ).

\section{COMMENT}

The distribution patterns of patent vs. non-patent primary entry tears after treatment warrant attention in detail. The majority of entry tears in patients undergoing surgery for acute type A aortic dissection are located in the proximal thoracic aorta and can therefore be addressed by surgical resection during repair [7]. Nevertheless, a certain percentage, being potentially underreported in the literature, do have their primary entry at a more distal site, such as the descending aorta with retrograde propagation of the dissecting membrane into the proximal thoracic aorta [8].This mechanism has also been confirmed in experimental studies [9]. In patients after surgery for acute type B aortic dissection, spontaneous closure of the primary entry tear is rare [10]. Consequently, as was also the case in our study, closure of the primary entry tear warrants any kind of surgical or interventional means [11]. As the current status of knowledge recommends TEVAR merely in patients with complicated type B aortic dissection-mainly due to malperfusion, a high percentage of patients with type $\mathrm{B}$ aortic dissection remain being treated by medical means and therefore do show a persisting patent entry [12].

The current classification defines a primary entry tear, multiple re-entries between lumina and a 'final' re-entry. We feel that for a better understanding of the natural history of the disease it would be more explanatory to classify the morphology as follows: 'primary entry tear, communications between lumina and re-entry'. From functional analyses from our group, which have not been published yet, there is evidence that the number and size of communications between lumina is decisive for diameter increase, in other words, the lower or smaller the communications between lumina, the more stable the natural course of the disease. Especially in these patients, closure of the primary entry tear is most effective in inducing a domino effect of readaption of the dissective membrane to the outer wall with the potential of full healing. However, if there are broad communications between both (or even more) lumina, closure of the primary entry tear may merely shift the problem distally, as the next broad communication between lumina may now functionally act as a primary entry tear and may therefore be causative for diameter increase. This is obviously the reason why many patients after successful TEVAR require type IV aneurysm repair years down the line, as the pathology mechanism has simply been shifted; the same is true for type A aortic dissection with an additional component of increased shear stress in the curvature of distal aortic arch. To summarize, this may explain the high number of 'patent primary entry tears'.

Furthermore, it is obvious-at least in proximal thoracic aortic segments-that the thrombosis status of the false lumen correlates with the status of the primary entry tear, as a fully thrombosed false lumen is unlikely in combination with a patent primary entry tear which has been demonstrated by our findings.

Survival during follow-up was favourable, which is at least partially attributable to our strict follow-up algorithm where patients are subjected to serial clinical and radiological follow-up visits on a yearly basis. As a consequence, progression of the remaining aortic disease can be diagnosed early enough to offer treatment by surgery or TEVAR. According to this algorithm, in 19\% of all patients, progression of the remaining underlying disease led to intervention by either surgery or TEVAR. Interestingly, there was a significant difference with regard to the need for intervention (surgery or TEVAR) during follow-up in the entire patient population with regard to the status of the primary entry tear. Patients with a patent primary entry tear had a significantly higher rate of any kind of intervention during the study period. One might argue that due to differences in age and comorbidities, the threshold for surgery or intervention might have been set differently. However, this was not the case. It is an interesting observation that patients sustaining dissections, irrespective of $A$ or B are hardly ever affected by other organ comorbidity. As such, any obliterative components of arteriopathy are extremely rare as is reduced ventricular function. In other words, the aortic pathology is the only limiting problem in the overwhelming majority of cases and therefore, the indication for secondary 
intervention or surgery is hardly ever affected by comorbidity or age, at least in our setting.

Interestingly, the status of the primary entry tear (patent vs. non-patent) had different implications with regard to the underlying thoracic aortic pathology. In patients after surgery for acute type A aortic dissection, a patent primary entry tear after treatment was significantly associated with a higher rate of intervention (surgery or TEVAR) during follow-up. Although the percentage of patients with a persisting patent primary entry tear after surgery for acute type A dissection was low, the number of patients requiring intervention of any kind along the thoracoabdominal aorta was impressively high. These findings underline the potential role of combined approaches such as the frozen elephant trunk procedure in order to treat the entire thoracic aortic disease to avoid late complications [13, 14]. Ongoing evaluations of these approaches will confirm their broader use in the future or will put them into question $[15,16]$.

Most interestingly, the status of the primary entry tear (patent vs. non-patent) was not associated with a higher rate of intervention (surgery or TEVAR) during follow-up in patients with acute type $B$ aortic dissection. This finding warrants further attention. Overall, the rate of intervention during follow-up in patients with an acute type B aortic dissection was, by far, higher than reported in the recent literature [17]. Several reasons may contribute to these findings, such as our algorithm of following-up these patients. Our numbers, with regard to survival and need for intervention (surgery or TEVAR), correlate inversely with the current literature; in other words, by a higher detection, we increase the number of patients treated and therefore we lower mortality in the chronic phase.

Multivariate regression analysis revealed a patent primary entry tear in patients after surgery for acute type A aortic dissection as the only independent predictor of intervention (surgery or TEVAR). These findings support the major importance of adequate imaging-if haemodynamics are favourable-in each and every patient with the diagnosis of an acute type A aortic dissection. Adequate imaging-in our opinion-contains a CTA of the entire aorta in order to perceive the extent of dissection, judge the involvement of branches and anticipate the repair strategy needed [7]. By determining exactly the site of the primary entry tear, a tear-oriented approach can be performed by conventional surgical means or by extending the armamentarium with a vascular and endovascular approach in order to be able to cover the primary entry tear as well as large communications between both lumina, which may have the same pathophysiological consequence as a patent primary entry tear, at least in the proximal and mid thoracic aorta [18]. Finally, if thoracoabdominal replacement is warranted distally in the future, surgery is eased as the arch, and the proximal and mid thoracic aortic segment are already fully treated [19].

Multivariate regression analysis did not reveal a patent primary entry tear as an independent predictor of intervention (surgery or TEVAR) during follow-up in patients after acute type B aortic dissection. This finding was unexpected and is contrary to the findings in patients after surgery of acute type A aortic dissection. Our explanations for this finding contain the fact that, despite full closure of the primary entry tear at the proximal thoracic level, communications in various numbers and sizes usually persist distally and may by themselves develop the same pathophysiological impact as a patent primary entry tear $[4,6]$. Overall, the number of interventions (surgery or TEVAR) in patients during follow-up after type $\mathrm{B}$ aortic dissection was impressively high and therefore will alert the aortic physician to keep these patients under close observation. But, it should also alert the entire community that up to $50 \%$ of all patients with a type B aortic dissection will require further invasive treatment as a result of continuing dilation of downstream segments, irrespective of whether TEVAR has been performed during the acute or early chronic phase.

Interestingly, the thrombosis status of the false lumen did not turn out to be an independent predictor of intervention (surgery or TEVAR) either during follow-up in patients after surgery for acute type A aortic dissection or during follow-up in patients with acute type $B$ aortic dissection. This is in contrast to recent findings from others [5]. However, the limitation in judging the thrombosis status of the false lumen may well be that the only means available is by comparing the enhancement of the false lumen with the true lumen. Moreover, in some situations, it remains speculative if the enhancement of the false lumen is in an antegrade fashion or in a retrograde fashion via distal communications with proximal runoff. Advances in imaging-most likely cine MRA-will add additional knowledge to this particular topic in the near future.

\section{Limitations and strengths of the study}

This study implies all the drawbacks of a retrospective analysis, especially with regard to imaging modalities in judging the thrombosis status of the false lumen. Functional imaging with regard to the dynamics of the dissection membrane and pressure conditions in both lumina may well be important surrogates that are not widely available yet and may have a bigger impact than morphologic findings. Furthermore, it has to be discussed whether a remaining type $B$ dissection after type $A$ repair is really comparable with a primary type $B$ aortic dissection. However, the idea to conduct the study with the current terminology and the current approach was from a clinical standpoint, as 'remaining type $B$ after type $A$ ' is the language clinicians use for surgery after type $A$ repair if a hemiarch replacement (which was the method of choice in the majority of patients in this study) has been used. It is clear that a short ascending replacement with residual dissection of the cranial ascending, the entire arch and all downstream segments represents a completely different entity and is not comparable with an isolated type B. However, extensive hemiarch replacement, which is the standard in our setting, merely leaves the supraaortic branches and the continuity between the left subclavian artery and the descending aorta as remaining native tissue. As such, the anatomic approximation to a 'remaining type $B$ after type $A$ ' is close. Finally, this study is the first of its kind demonstrating significant differences in the natural course of remaining type $B$ dissections after type $A$ repair and primary type $B$ dissections and may thereby serve as a basis for future investigations in this rapidly evolving field of knowledge.

To summarize, a remaining type B dissection after type A repair and a primary type $B$ aortic dissection represent two distinct pathophysiological entities with regard to the late outcome. The need for any kind of surgical reintervention in the thoracoabdominal aorta is significantly higher in primary type $B$ aortic dissections. A remaining patent primary entry tear independently predicts the need for intervention (surgery or TEVAR) in patients after surgery for acute type A aortic dissection and therefore remains the main target of initial therapy. The 
thrombosis status of the false lumen seems to be of secondary importance.

\section{Conflict of interest: none declared.}

\section{REFERENCES}

[1] Fattori R, Bacchi-Reggiani L, Bertaccini P, Napoli G, Fusco F, Longo M et al. Evolution of aortic dissection after surgical repair. Am J Cardiol 2000;86:868-72.

[2] Barron DJ, Livesey SA, Brown IW, Delaney DJ, Lamb RK, Monro JL. Twenty-year follow-up of acute type a dissection: the incidence and extent of distal aortic disease using magnetic resonance imaging. J Card Surg 1997;12:147-59.

[3] Coselli JS, Bozinovski J, LeMaire SA. Open surgical repair of 2286 thoracoabdominal aortic aneurysms. Ann Thorac Surg 2007;83:S862-4.

[4] Schoder M, Czerny M, Cejna M, Rand T, Stadler A, Sodeck GH et al. Endovascular repair of acute type $B$ aortic dissection: long-term followup of true and false lumen diameter changes. Ann Thorac Surg 2007;83: 1059-66.

[5] Tsai TT, Evangelista A, Nienaber CA, Myrmel T, Meinhardt G, Cooper JV et al. International Registry of Acute Aortic Dissection. Partial thrombosis of the false lumen in patients with acute type B aortic dissection. $N$ Engl Med 2007;357:349-59.

[6] Song JM, Kim SD, Kim JH, Kim MJ, Kang DH, Seo JB et al. Long-term predictors of descending aorta aneurysmal change in patients with aortic dissection. J Am Coll Cardiol 2007;50:799-804.

[7] Czerny M, Krähenbühl E, Reineke D, Sodeck G, Englberger L, Weber A et al. Mortality and neurologic injury after surgical repair with hypothermic circulatory arrest in acute and chronic proximal thoracic aortic pathology: effect of age on outcome. Circulation 2011;124:1407-13.

[8] Sun L, Qi R, Chang Q, Zhu J, Liu Y, Yu C et al. Surgery for acute type A dissection with the tear in the descending aorta using a stented elephant trunk procedure. Ann Thorac Surg 2009;87:1177-80.

[9] Dziodzio T, Juraszek A, Reineke D, Jenni H, Zermatten E, Zimpfer $D$ et al. Experimental acute type $\mathrm{B}$ aortic dissection: different sites of primary entry tears cause different ways of propagation. Ann Thorac Surg 2011; 91:724-7.

[10] Bernard Y, Zimmermann H, Chocron S, Litzler JF, Kastler B, Etievent JP et al. False lumen patency as a predictor of late outcome in aortic dissection. Am J Cardiol 2001;87:1378-82.

[11] Hutschala D, Fleck T, Czerny M, Ehrlich M, Schoder M, Lammer J et al. Endoluminal stent-graft placement in patients with acute aortic dissection type B. Eur J Cardiothorac Surg 2002;21:964-9.

[12] Nienaber CA, Rousseau H, Eggebrecht H, Kische S, Fattori R, Rehders TC et al. INSTEAD Trial. Randomized comparison of strategies for type B aortic dissection: the INvestigation of STEnt Grafts in Aortic Dissection (INSTEAD) trial. Circulation 2009;120:2519-28.

[13] Tsagakis K, Pacini D, Di Bartolomeo R, Gorlitzer M, Weiss G, Grabenwoger $\mathrm{M}$ et al. Multicenter early experience with extended aortic repair in acute aortic dissection: Is simultaneous descending stent grafting justified? J Thorac Cardiovasc Surg 2010;140(6 Suppl.):S116-20.

[14] Tsagakis K, Pacini D, Di Bartolomeo R, Benedik J, Cerny S, Gorlitzer M et al. Arch replacement and downstream stent grafting in complex aortic dissection: first results of an international registry. Eur J Cardiothorac Surg 2011;39:87-93; discussion 93-4.

[15] Gorlitzer M, Weiss G, Thalmann M, Mertikian G, Wislocki W, Meinhart J et al. Combined surgical and endovascular repair of complex aortic pathologies with a new hybrid prosthesis. Ann Thorac Surg 2007;84: 1971-6.

[16] Shimamura K, Kuratani T, Matsumiya G, Kato M, Shirakawa Y, Takano H et al. Long-term results of the open stent-grafting technique for extended aortic arch disease. J Thorac Cardiovasc Surg 2008;135:1261-9.

[17] Parsa CJ, Schroder JN, Daneshmand MA, McCann RL, Hughes GC. Midterm results for endovascular repair of complicated acute and chronic type B aortic dissection. Ann Thorac Surg 2010;89:97-102.

[18] Uchida N, Katayama A, Tamura K, Sutoh M, Kuraoka M, Ishihara $H$. Frozen elephant trunk technique and partial remodeling for acute type A aortic dissection. Eur J Cardiothorac Surg 2011:40:1066-71.

[19] Dumfarth J, Michel M, Schmidli J, Sodeck G, Ehrlich M, Grimm M et al. Mechanisms of failure and outcome of secondary surgical interventions after thoracic endovascular aortic repair (TEVAR). Ann Thorac Surg 2011; 91:1141-6

\section{APPENDIX. CONFERENCE DISCUSSION}

Dr C. Mestres (Barcelona, Spain): In this study, Dr Roost and colleagues analysed the outcome and the need for surgical intervention in patients after remaining type $B$ dissection after type $A$ repair, and primary type B aortic dissection. This is an exciting area of work because little, if any, has been done previously as they have correctly stated. Their experience with 359 patients shows interesting data. First, if patients are appropriately followed after discharge, the rate of late reintervention based on aortic dilatation is significant, roughly $20 \%$ at a median of a very short follow-up of 24 months. This to me stresses the need for following the patients well. This has been our policy over the years, and we agree with the authors.

Second, a patent tear after type A predicts late reintervention, but this is not the case after primary type B dissection. Third, and very interesting to me, the different degrees of thrombosis of the false lumen do not predict the need for late reintervention. And, finally, the need for late reintervention is far higher in the case of primary type B dissection.

Furthermore, I believe that this study confirms our longstanding suspicion that type $A$ and type $B$ dissection are different diseases, based on the late behaviour of these patients, and this is confirmed by the different requirements for reintervention over time. This study to me is also important considering the dynamic behaviour of the aorta after any major proximal or distal event. Based on your data, I wanted to ask you the following two questions, and you can reply, of course, sequentially.

First, as we know that the treatment of the primary tear is of paramount importance in acute type A dissection, and that we have to count on distal re-entries as well, do you think that the frozen elephant trunk approach might be mandatory in the future in this disease to entirely treat the intrathoracic aorta?

Dr Roost: Well, you just published data which actually proves that is the case. I think if you look at the whole picture in patients with type A aortic dissection, the primary thing is to have a surviving patient. So to make a very complicated situation more complicated, in primary intervention you have to just keep in mind that the first thing should be that it does not affect your early mortality. But I do think that if you have a patient with a type A aortic dissection, you have to have really good imaging done prior to your first operation and just take your time and really assess the anatomical situation. And let's say if you are a good surgeon and you know that you will not affect your mortality by using a frozen elephant technique and replacing the whole aortic arch, then I think, yes, you should do it if you have an entry further down in the downstream aorta.

Dr Mestres: The second question is probably a more provocative one, and it is, do you think that acute complicated type B dissection should be treated from the front, as then the risk of retrograde extension and proximal endoleaks of stent grafts would be eliminated? This would also mean a full treatment of the entire thoracic aorta.

Dr Roost: In acute complicated type B, it is a difficult question because it is a lot of surgery to do in the acute situation. Our data shows that the mortality is low for type B aortic dissection, and so I am a little bit hesitant to say, yes, sure, you should do that, keeping in mind that you have a low mortality and you may risk that if you have a lot of surgery in the acute phase. But we have to look a lot more at how we can prevent that. Almost $30 \%$ of patients after primary type B aortic dissection need open thoraco-abdominal replacement and that has a huge impact, but I cannot answer you exactly.

Dr Mestres: It was just provocative anyway.

Dr A. Rajaii-Khorasani (Mashhad, Iran): There was a very famous actor in the United States by the name of Lucille Ball. Lucille died with the same issue, an acute dissection, operated at Cedars-Sinai, and then died from rupture of the untreated aorta; around the same time, I had a patient who died with the same. So I want to stress the importance of follow-up of patients with type A dissection who have had successful surgery, and who have gone on to have type $\mathrm{B}$ dissection during the first month after the event. And if you see an area of the aorta that is getting larger, it has to be treated; otherwise, these are the ones who will rupture.

Dr Roost: I totally agree.

Dr S. Trimarchi (San Donato Milanese, Italy): In your last answer to $\mathrm{Dr}$ Mestres about the long-term follow-up both for type A and type B, you mentioned that $30 \%$, one-third, of these patients can develop a thoraco-abdominal aneurysm. I think all of us know that you are developing many types of imaging signs in order to predict this in the future. So would you tell us something more about this topic, not only regarding the proximal patency of straight versus spiral, or the role of the diameter of the false lumen. I mean, have you collected all this information in order to put it all together and give us something more? 
Dr Roost: That is an ongoing project. One limitation of that study is that it is CT based and you all know that the technology, fortunately, evolves very quickly, and maybe also one reason why the thrombosis status of the false lumen did not predict outcome was that it was sometimes actually hard to say whether it was fully or partially thrombosed. So I think we should probably use more MR scans because it is a dynamic process, and we are actually continuing to collect this kind of data, yes, but I cannot give you any results yet.

Dr B. Zipfel (Berlin, Germany): The main message is, in type A dissection, you should not leave an open entry behind, which is usually in the descending aorta. I have a question on the type B group. These were native dissections, right?

Dr Roost: Yes.

Dr Zipfel: Non-stented dissections?

Dr Roost: Some were stented in the initial phase, and we saw that even if you treat them with a stent in the initial phase, you still cannot rule out that they do not have a progressive dilatation in their thoraco-abdominal aorta. We have a few cases which actually had to have an open surgery after initial therapy with the stent graft.

Dr Zipfel: Okay. You should mention this. I was surprised how many entries in type B dissection had occluded spontaneously. I have never seen this except in very rare cases. In how many patients did the entries close, and how many had a stent graft put in of those in whom entries closed?

Dr Roost: You are right, we have to state and we probably have to include that in our work. I cannot tell you exactly what the percentages were, but the fact remains, that is what I said before, that a closed entry in type B aortic dissection does not tell you whether there is a reintervention or not. And that was actually surprising for us. And I think maybe one reason is that you more often do not only have one entry but a lot of communications, and even if you close the first primary entry, you still have communications farther down which results in an aneurysm expansion. 